\title{
Conserved and non-conserved functions of the rice homologs of the Arabidopsis trichome initiation-regulating MBW complex proteins
}

Kaijie Zheng ${ }^{1,2+}$, Xutong Wang ${ }^{2 \dagger}$, Yating Wang ${ }^{2}$ and Shucai Wang ${ }^{2,3^{*}}$

\begin{abstract}
Background: Trichome initiation in Arabidopsis is regulated by a MYB-bHLH-WD40 (MBW) transcriptional activator complex formed by the R2R3 MYB transcription factor GLABRA1 (GL1), MYB23 or MYB82, the bHLH transcription factor GLABRA3 (GL3), ENHANCER OF GLABRA3 (EGL3) or TRANSPARENTTESTA8 (TT8), and the WD40-repeat protein TRANSPARENT TESTA GLABRA1 (TTG1). However, the functions of the rice homologs of the MBW complex proteins remained uncharacterized.
\end{abstract}

Results: Based on amino acid sequence identity and similarity, and protein interaction prediction, we identified OsGL1s, OsGL3s and OsTTG1s as rice homologs of the MBW complex proteins. By using protoplast transfection, we show that OsGL1D, OsGL1E, OsGL3B and OsTTG1A were predominantly localized in the nucleus, OsGL3B functions as a transcriptional activator and is able to interact with GL1 and TTG1. By using yeast two-hybrid and protoplast transfection assays, we show that OsGL3B is able to interact with OsGL1E and OsTTG1A, and OsGL1E and OsTTG1A are also able to interact with GL3. On the other hand, we found that OsGL1D functions as a transcription activator, and it can interact with GL3 but not OsGL3B. Furthermore, our results show that expression of OsTTG1A in the $\operatorname{ttg} 1$ mutant restored the phenotypes including alternations in trichome and root hair formation, seed color, mucilage production and anthocyanin biosynthesis, indicating that OSTTG1A and TTG1 may have similar functions.

Conclusion: These results suggest that the rice homologs of the Arabidopsis MBW complex proteins are able to form MBW complexes, but may have conserved and non-conserved functions.

Keywords: MBW complex, Trichome initiation, Transcription factor, Rice, Arabidopsis

\section{Background}

Trichomes are appendages on the surfaces of the aerial parts of the plants. Trichomes are developed from epidermal cells and are diverse in appearance. Trichomes

\footnotetext{
*Correspondence: wangshucai@yahoo.com
}

${ }^{\dagger}$ Kaijie Zheng and Xutong Wang contributed equally to this work.

${ }^{2}$ Key Laboratory of Molecular Epigenetics of MOE, Institute of Genetics

and Cytology, Northeast Normal University, Changchun, China

Full list of author information is available at the end of the article can protect plants from excessive heat and water loss, and from insect or pathogen attacks by increasing the boundary layer thickness between the epidermal tissues and the environment $[1,2]$.

Available evidence suggests that trichome initiation in Arabidopsis is regulated by a MYB-bHLH-WD40 (MBW) complex formed by a R2R3 MYB transcriptional activator, a bHLH transcription factor, and a WD40-repeat protein [3-7]. The R2R3 MYB transcription factor in this MBW complex is GLABRA1 (GL1) [8], the bHLH transcription factor is GLABRA3 (GL3), ENHANCER OF 
GLABRA3 (EGL3) $[9,10]$, or TRANSPARENT TESTA8 (TT8) [11], and the WD40-repeat protein is TRANSPARENT TESTA GLABRA1 (TTG1) [12]. It has been shown that MYB23 and MYB82 are also able to interact with GL3 and/or EGL3, and to regulate trichome formation [13, 14]. The MBW transcriptional activator complex is able to induce the expression of the homeodomain protein gene GLABRA2 (GL2) [15], leading to the promotion of trichome initiation $[3-7,16]$.

This MBW complex is also able to induce the expression of some R3 MYB genes including TRYPTICHON (TRY), CAPRICE (CPC), ENHANCER OF TRY AND CPC1 (ETC1) and ETC3 [17-22]. These R3 MYB transcriptional factors, including ETC2, TRICHOMELESS1 (TCL1) and TCL2, whose expression are not regulated by the MBW complex [22-25], are able to move to their neighboring cells, where they competing with GL1 for binding of GL3, therefore inhibiting the formation of the MBW complex, leading to the inhibition of trichome initiation [3-7, 26-28].

At least in some plants, functions of MBW complex proteins in regulating trichome initiation is conserved, for example, Brassica napus plants expressing Arabidopsis GL3 produced ectopic trichomes [29], cotton homologs of GL1 and GL2 regulate trichome initiation in Arabidopsis [30, 31], and trichome phenotypes in the ttg1 mutants were restored by expressing an apple TTG1 homolog gene [32].

Even though trichomes could provide protection for plants [1, 2], glabrous has been considered to be a favorite agronomic trait in rice (Oryza sativa), because grains of glabrous rice have greater packing capability, and glabrous rice produces less itchy causing dust [33, 34]. Therefore, great efforts have been devoted to addressing the regulation mechanisms under the control of trichome initiation in rice.

So far, several regulator of trichome initiation have been identified in rice, some of them are homologs of Arabidopsis trichome initiation regulators, whereas others are not. For example, OsWOX3B, a homolog of GL2, regulates trichome initiation in rice [33-35]. SPL9 is a squamosa promoter binding type protein that has been shown to regulate trichome initiation in Arabidopsis via directly regulating the expression of TCL1 [36], OsSPL10, a homolog of SPL9 is also able to regulate trichome initiation in rice [37]. These results suggest that trichome initiation in rice may be regulated by similar mechanisms as in Arabidopsis.

On the other hand, SDG714, a histone H3K9 Methyltransferase, HL6 (Hairy Leaf 6), an AP2/ERF transcription factor and type- $\mathrm{B}$ response regulators have been shown to be involved in the regulation of trichome initiation in rice [35, 38, 39], but none of them are homologs of known Arabidopsis trichome initiation regulators. Our previous studies have also shown that OsTCL1, a homolog of TCL1, is able to regulate trichome initiation in Arabidopsis, but not in rice [40]. These results indicate that trichome initiation in rice may also be regulated by different mechanisms.

Here we report the identification and characterization of rice homologs of the Arabidopsis trichome initiationregulating MBW complex proteins. Based on amino acid sequence identity and similarity, and protein interaction prediction, we identified OsGL1A-OsGL1E, OsGL3AOsGL3C, and OsTTG1A and OsTTG1B as homologs of GL1, GL3 and TTG1, respectively. By using Arabidopsis protoplast transfection assays, we found that these proteins may have conserved and non-conserved functions in forming MBW complexes. By generating transgenic plants expressing OsTTG1A in the ttg1 mutants, we show that OsTTG1A and TTG1 may have similar functions in regulating trichome initiation as well as root hair formation and secondary metabolism in Arabidopsis.

\section{Results \\ Homologs of the MBW complex proteins in rice}

In previous experiments, we have identified OsGL1A, OsGL1B and OsGL1C as rice homologs of Arabidopsis GL1, OsGL3A, OsGL3B and as rice homologs of Arabidopsis GL3, and OsTTG1A, and OsTTG1B as rice homologs of Arabidopsis TTG1[40].

To examine if these MBW homologs in rice can form MBW complexes, we first analyzed their interaction relationship on STRING (https://string-db.org/). We found that OsGL1D (Loc_Os03g29614) and OsGL1E (Loc_ Os06g10350) were predicted as potential interaction proteins of OsGL3B. As shown in Fig. 1a, OsGL1 proteins showed a $29.4 \% \sim 36.3 \%$ identity, and a $43.9 \% \sim 54.6 \%$ similarity with GL1 at amino acid level (Fig. 1a). Phylogenetic analysis showed that OsGL1A is closely related to OsGL1B, whereas OsGL1D is closed related to OsGL1E. Together with OsGL1C, these five OsGL1s formed a clade (Fig. 1b). On the other hand, GL1 is closed related to MYB23, and they formed another clade together with MYB82 (Fig. 1b). Sequence alignment showed that the most conserved region of the OsGL1s is the R2R3 MYB domain (Fig. S1). The $[\mathrm{D} / \mathrm{E}] \mathrm{L} \times 2[\mathrm{R} / \mathrm{K}] \times 3 \mathrm{~L} \times 6 \mathrm{~L} \times 3 \mathrm{R}$ amino acid signature required for the interaction of MYB transcription factors with R/B-like bHLH transcription factors [41], and the $S$ residue has been shown to be required for the activation of GL2 [42], are fully conserved in all the five OsGL1s (Fig. S1).

As for the OsGL3s, both OsGL3A and OsGL3B showed a more than $33 \%$ identity and a more than $51 \%$ similarity with GL3 and EGL3, whereas that for OsGL3C are only about $25 \%$ and $40 \%$, respectively 
A

\begin{tabular}{|c|c|c|c|c|c|c|}
\hline & GL1 & $\overline{\text { OsGL1A }}$ & OsGL1B & OsGL1C & OsGL1D & OSGL1E \\
\hline GL1 & & $\overline{35.6}$ & $\overline{34.2}$ & 36.3 & 29.4 & 32.2 \\
\hline OsGL1A & 50.6 & & 66.3 & 37.8 & 36.6 & 39.3 \\
\hline OsGL1B & 54.6 & 78.5 & & 37.5 & 36.4 & 40.8 \\
\hline OsGL1C & 49.5 & 53.1 & 53.5 & & 39.0 & 38.3 \\
\hline OsGL1D & 43.9 & 48.3 & 49.2 & 50.8 & & 41.4 \\
\hline OsGL1E & 49.4 & 51.3 & 56.1 & 54.2 & 51.7 & \\
\hline
\end{tabular}

B

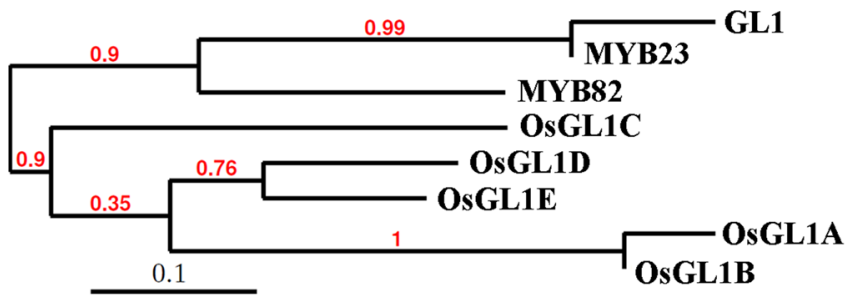

C

\begin{tabular}{|c|c|c|c|c|c|}
\hline & GL3 & EGL3 & $\overline{\text { OsGL3A }}$ & OsGL3B & $\overline{\text { OsGL3C }}$ \\
\hline$\overline{\text { GL3 }}$ & & $\overline{74.4}$ & $\overline{34.2}$ & 33.3 & $\overline{24.4}$ \\
\hline EGL3 & 83.8 & & 34.6 & 36.0 & 25.6 \\
\hline OsGL3A & 51.5 & 54.0 & & 56.7 & 23.4 \\
\hline OsGL3B & 51.2 & 54.7 & 70.6 & & 24.7 \\
\hline OsGL3C & 38.0 & 41.1 & 39.0 & 40.6 & \\
\hline
\end{tabular}

D

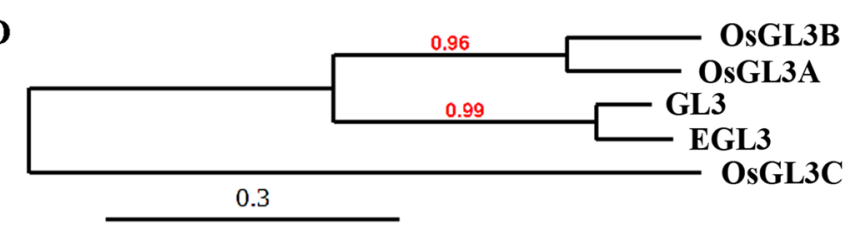

$\mathbf{E}$

\begin{tabular}{cccc}
\hline \multicolumn{4}{c}{ TTG1 OsTTG1A OsTTG1B } \\
\hline TTG1 & & 60.5 & 49.3 \\
OsTTG1A & 75.4 & & 47.4 \\
OsTTG1B & 63.7 & 60.1 & \\
\hline
\end{tabular}

F

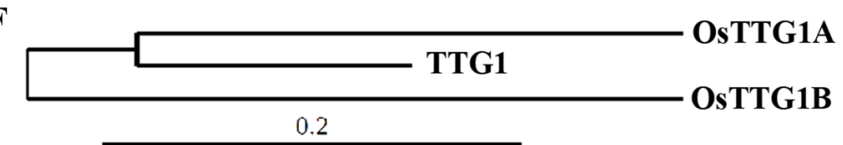

Fig. 1 GL1, GL3 and TTG1 homologs in rice. a Amino acid identity and similarity of GL1 and OsGL1s. OsGL1s, homologs of GL1 were identified by using "Protein Homologs" on Phytozome (https://phytozome.jgi.doe.gov/pz/portal.html\#). Percentages of amino acid similarity and identity of GL1 and OsGL1s were calculated by using MatGAT (v2.02). Percentage of amino acid identity are shaded in green, and percentage of amino acid similarity in blue. $\mathbf{b}$ Phylogenetic tree of GL1 and OsGL1s. The entire amino acid sequences of GL1 and OsGL1s were used for phylogenetic analysis on Phylogeny (www.phylogeny.fr) by using "One Click" mode with default settings. The number above the branch indicates branch support values. Bar indicates branch length. c Amino acid identity and similarity of GL3 and OsGL3s. OsGL3s were identified by using "Protein Homologs" on Phytozome (https://phytozome.jgi.doe.gov/pz/portal.html\#). Percentages of amino acid similarity and identity of GL3 and OsGL3s were calculated by using MatGAT (v2.02). Percentage of amino acid identity are shaded in green, and percentage of amino acid similarity in blue. $\mathbf{d}$ Phylogenetic tree of GL3 and OsGL3s. The entire amino acid sequences of GL3 and OsGL3s were used for phylogenetic analysis on Phylogeny (www.phylogeny. fr) by using "One Click" mode with default settings. The number above the branch indicates branch support values. Bar indicates branch length. e Amino acid identity and similarity of TTG1 and OsTTG1s. OsTTG1s were identified by using "Protein Homologs" on Phytozome (https://phytozome. jgi.doe.gov/pz/portal.html\#). Percentages of amino acid similarity and identity of TTG1 and OsTTG1s were calculated by using MatGAT (v2.02). Percentages of amino acid identity are shaded in green, and percentage of amino acid similarity in blue. f Phylogenetic tree of TTG1 and OsTTG1s. The entire amino acid sequences of TTG1 and OsTTG1s were used for phylogenetic analysis on Phylogeny (www.phylogeny.fr) by using "One Click" mode with default settings. The number above the branch indicates branch support values. Bar indicates branch length 
(Fig. 1c). Phylogenetic analysis showed that OsGL3A is closely related to OsGL3B, and they formed a clade with GL3 and EGL3 pair (Fig. 1d). Sequence alignment showed that the most conserved regions of the OsGL3s are the $\mathrm{N}$-terminal and $\mathrm{C}$-terminal domains (Fig. S2). OsGL3A and OsGL3B, but not OsGL3C showed high similarity to GL3 and EGL3 at the HLH domain region and the first 97 amino acids required for GL3 to interact with GL1 [9] (Fig. S2).

Among the MBW complex protein homologs in rice, OsTTG1s are the most conserved ones when compared with their Arabidopsis homologs. OsTTG1A and OsTTG1B showed a $60.5 \%$ and $49.3 \%$ identity, and a $75.4 \%$ and $63.7 \%$ similarity, respectively to TTG1 (Fig. 1e). Phylogenetic analysis showed that OsTTG1A is closely related to TTG1 (Fig. 1f). Sequence alignment showed OsTTG1s and TTG1 are highly conserved at full-length amino acid sequence level (Fig. S3), including the 25 amino acid sequence that is required for interaction of TTG1 with GL3 [9].

In order to get a better pictures on the relations that exists between the Arabidopsis MBW complex component proteins and their rice homologs, we identified MBW complex component protein homologs, i.e., proteins with highest amino acid similarity with GL1, GL3 and TTG1, respectively, in the Brassicaceae family plants Brassica rapa, Capsella grandiflora and Capsella rubella, the Fabidae family plant Glycine max, the Malpighiales family plant Populus trichocarpa, and the Panicoideae family plants Zea mays, Setaria italica and Panicum hallii, and expended the phylogenetic analysis. The results show that OsGL1s and the Arabidopsis GL1, MYB23 and MYB82 are still in two different clades (Fig. S4). The Arabidopsis GL1, MYB23 and MYB82 are closely related to homologs from the three Brassicaceae plants and the Malpighiales family plant P. trichocarpa, whereas OsGL1s are closely related to homologs from the three Panicoideae family plants and the Fabidae family plant G. $\max$ (Fig. S4). On the other hand, OsGL3C and TT8 formed a clade, whereas GL3, EGL3, OsGL3A and OsGL3B formed another clade with homologs from all the eight plants mentioned above, in which OsGL3A and OsGL3B formed a sub-clade with homologs from the three Panicoideae family plants, and GL3 and EGL3 formed another sub-clade with homologs from the three Brassicaceae plants, P. trichocarpa and G. $\max$ (Fig. S5). For the WD40 proteins, OsTTG1B alone formed a clade, whereas OsTTG1A, TTG1 and homologs from all the eight plants formed another clade, in which the OsTTG1A and homologs from the three Panicoideae family plants formed a sub-clade, and TTG1 and homologs from the three Brassicaceae plants,
P. trichocarpa and G. max formed another sub-clade (Fig. S6).

\section{Subcellular localization of the MBW complex homolog proteins}

Previous reports have shown that GL3, GL1 and TTG1 are all localized in the nucleus [43]. Based on the above bioinformatics analysis, OsGL1A, OsGL1B, OsGL1C, OsGL1D, OsGL1E, OsGL3B and OsTTG1A were chosen for subcellular localization assays. OsGL1A and OsGL1B were chosen because they showed relatively high amino acid identity and similarity to GL1. Whereas OsGL1D and OsGL1E are potential interactors of OsGL3B according to STRING assays. OsGL3B was chosen because both OsGL3A and OsGL3B showed relatively high amino acid identity and similarity to GL3, OsGL3B was predicted to interact with OsGL1D and OsGL1E on STRING, whereas OsGL1C is not paired with other OsGL1 proteins. OsTTG1A was chosen because it showed relatively high amino acid identity and similarity to TTG1.

We examined their subcellular localization in Arabidopsis protoplasts. GFP fused constructs of the MBW complex homolog genes were transfected into Arabidopsis protoplasts, and GFP fluorescence was observed under a confocal microscope. We found OsGL1D, OsGL1E, OsGL3B and OsTTG1A were predominantly localized in nucleus, whereas OsGL1A and OsGL1B may be localized in nucleus and likely some other organelles such as cell membranes and chloroplasts (Fig. 2).

\section{OsGL3B is a transcriptional activator and it interacts with GL1 and TTG1 in Arabidopsis protoplasts}

We have previously shown that GL3 functions as a transcription activator in transfected Arabidopsis protoplasts [44]. To examine if the MBW complex homologs in rice can indeed form MBW complexes, we examined if OsGL3B may also functions as a transcription activator. Plasmids of effector gene GD, GD-OsGL3B or $G D$ GL3, together with the reporter gene Gal4-GUS were co-transfected into Arabidopsis protoplasts, and GUS activities were examined by using a microplate reader. The results show that, similar to GD-GL3, cotransfection of $G D-O s G L 3 B$ activated the reporter gene expression (Fig. 3a).

Having shown that OsGL3B functions as a transcriptional activator, we examined if OsGL3B may form a MBW complex with GL1 and TTG1 by examining their interactions in yeast cells and Arabidopsis protoplasts. As shown in Fig. 4, OsGL3B interacted with GL1 and TTG1 in yeast cells. Cotransfection of OsGL3B with $G D-G L 1$ and GD-TTG1, respectively activated reporter 


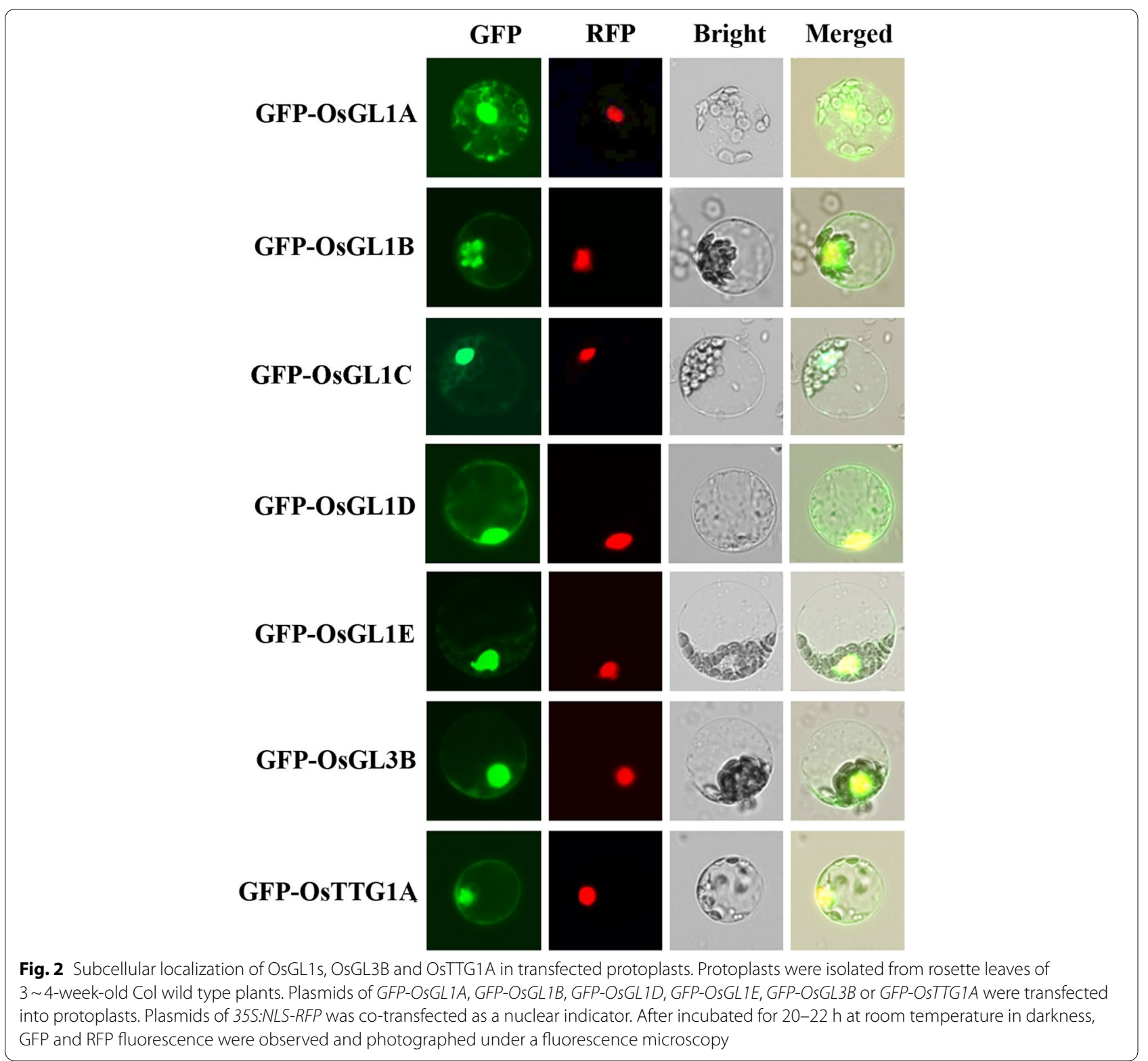

gene expression in protoplasts (Fig. 3b), indicating that OsGL3B may be able to interact with GL1 and TTG1 in plant cells.

\section{Interactions of OsTTG1A and OsGL1s with OsGL3B and GL3}

The above results suggest that OsGL3B is able to form a MBW complex with GL1 and TTG1. We then further examined if it may form MBW complex with OsGL1s and OsTTG1A. To do that, we examined interaction of OsGL3B with OsGL1s and OsTTG1A in yeast cells and Arabidopsis protoplasts. As shown in Fig. 4, OsGL3B is able to interact with OsGL1D, OsGL1E and OsTTG1.
Similarly, cotransfection of OsGL3B with GD-OsTTG1 activated reporter gene expression in protoplasts, whereas cotransfection of OsGL3B with GD-OsGL1A or $G D-O s G L 1 B$ failed to do so (Fig. 5a). However, cotransfection of OsGL3B with GD-OsGL1E activated reporter gene expression (Fig. 5b). These results suggest that OsGL1E, OsGL3B and OsTTG1A can form a MBW complex.

Our protoplast transfection assays also suggest that both OsTTG1A and OsGL1E can interact with GL3 (Fig. 5), indicating that MBW complex proteins in Arabidopsis and rice are interchangeable in forming $\mathrm{MBW}$ complex. 

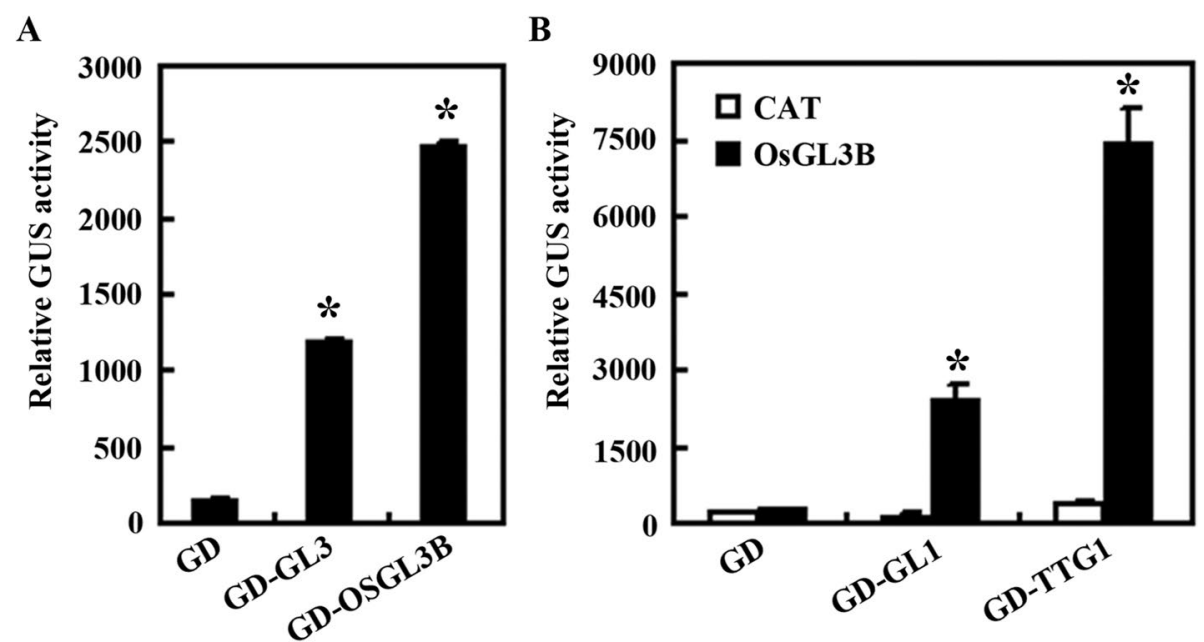

Fig. 3 OsGL3B is a transcriptional activator, and it interacts with GL1 and TTG1 in transfected protoplasts. a OsGL3B is a transcriptional activator. Plasmids of the Gal4:GUS reporter, and GD-OsGL3B or GD-GL3 effector were co-transfected into protoplasts. Cotransfection of the plasmids of GD effector was used as a control. After incubated for 20-22 h at room temperature in darkness, GUS activities were measured by using a microplate reader. Data represent the mean \pm SD of three biological replicates. *: Significantly different from the GD control (Student's $t$-test, $P<0.05$ ). b OsGL3B is able to interact with GL1 and TTG1. Plasmids of the Gal4:GUS reporter, GD-GL1 or GD-TTG1, and OsGL3B or CAT effectors were co-transfected into protoplasts. Cotransfection of the plasmids of GD effector was used as a control. After incubated for 20-22 h at room temperature in darkness, GUS activities were measured by using a microplate reader. Data represent the mean \pm SD of three biological replicates. *: Significantly different from the CAT control (Student's t-test $P<0.05)$

Surprisingly, we found that transfection of $G D$ OsGL1D activated reporter gene expression (Fig. 5b), suggesting that unlike GL1 and other OsGL1s examined, OsGL1D functions as a transcription activator. Our results also show that GUS activities were increased when $G L 3$, but not $O s G L 3 B$ was cotransfected with $G D$ OsGL1D (Fig. 5b), indicating that OsGL1D is able to interact with GL3, but not OsGL3B.

\section{Ectopic expression of OsTTG1A rescued $t$ tg 1 phenotypes}

After showing that OsGL1E, OsGL3B and OsTTG1A can form a MBW complex, we wanted to further examine if they may have similar functions as their Arabidopsis homologs. Considering that the ttg1 mutant has a variety of obvious phenotypes relate to trichome and root hair cell fate determination and secondary metabolism including seed color, mucilage production and anthocyanin biosynthesis [12, 45, 46], and OsTTG1A showed high amino acid identity and similarity to TTG1, we decided to examine if OsTTG1A is a functional analogue of TTG1 by examine if ectopic expression of OsTTG1A could rescue the ttg1 mutant phenotypes.

Transgenic plants were generated in the $\operatorname{tg} 1$ mutant plants by expressing OsTTG1A under the control of the $35 S$ promoter (35S:OsTTG1A/ttg1). Two independent homozygous lines were used for phenotypic analysis. As shown in Fig. 6a, transcript of TTG1 was only detectable in the Ler wild type plants, whereas transcript of
OsTTG1A was only detectable in the 35S:OsTTG1A/ ttg1 transgenic plants, and relative high transcript level of OsTTG1A was observed in seedlings of the 35S:OsTTG1A/ttg1 \#1 line. We observed that plants of both 35S:OsTTG1A/ttg1 transgenic lines produced trichomes on rosette leaves and stems (Fig. 6b). Quantitative analysis showed that plants of the 35S:OsTTG1A/ ttg1 \#1 line produced more trichomes on rosette leaves (Fig. 6c), consistent with the relatively high transcript level in seedlings of this line. On the other hand, reduced root hairs formation was observed in both of the 35S:OsTTG1A/ttg1 transgenic lines when compared with the ttg1 mutants (Fig. 7a), and quantitative analysis showed that root hair density in the 35S:OsTTG1A/ ttg1 transgenic seedlings is similar to the Ler wild type (Fig. 7b).

The seed color phenotype of the ttg1 mutant was recovered in the 35S:OsTTG1A/ttg1 transgenic plants, but also to different degree in the two different lines (Fig. 8a). Whereas mucilage production in the 35S:OsTTG1A/ttg1 \#1 line was nearly similar to the Ler wild type, but that in \#2 line was largely similar to the ttg1 mutants (Fig. 8b), anthocyanin biosynthesis was also largely recovered in the 35S:OsTTG1A/ttg1 \#1 line, but not \#1 line seedlings (Fig. 8c). These results indicate that OsTTG1A is likely the functional analogue of TTG1.

We then examined the expression of the TTG1 down stream trichome formation regulator genes in the Ler 


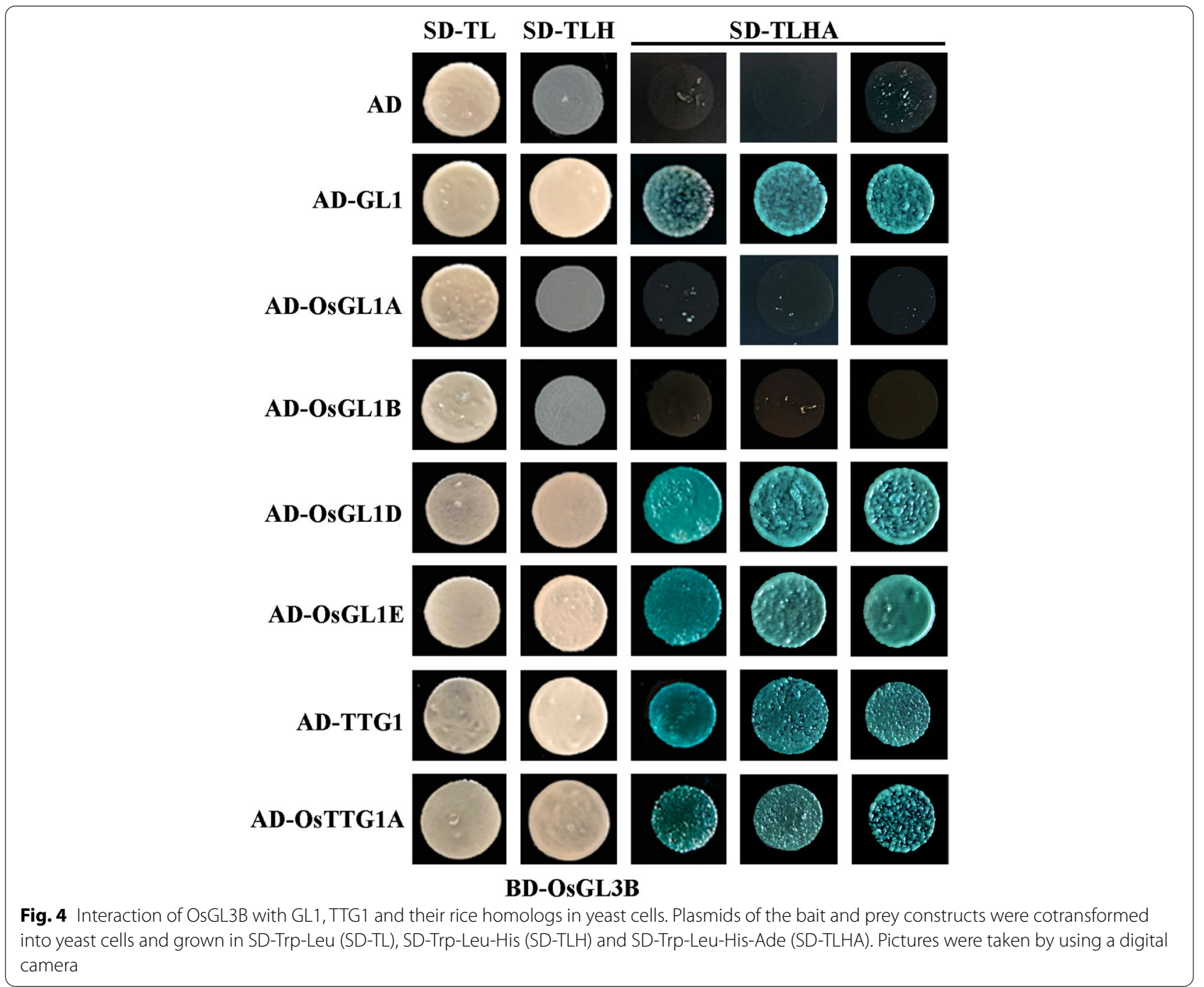

wild type, the ttg1 mutant and 35S:OsTTG1A/ttg1 transgenic plant seedlings, including GL2 and the R3 MYB genes. We found that the expression level of GL2 was significantly reduced, whereas that of ETC2 and TCL2 increased in the ttg1 mutants, whereas that in the 35S:OsTTG1A/ttg1 transgenic plants were largely similar to the Ler wild type seedlings (Fig. 9).

\section{Discussion}

A MYB-bHLH-WD40 (MBW) complex formed by the R2R3 MYB transcriptional activator GL1, the bHLH transcription factor GL3, EGL3 or TT8, and the WD40repeat protein TTG1 regulates trichome initiation in Arabidopsis [3, 4, 6, 7, 24]. By identifying and characterizing rice homologs of the trichome initiation-regulating MBW complex proteins, we found that similar MBW complex may present in rice and at least some components in the complex may have similar functions as the ones in Arabidopsis.

First, the rice homologs shared similar features as the Arabidopsis MBW complex, i.e., the $[D / E]$ $\mathrm{L} \times 2[\mathrm{R} / \mathrm{K}] \times 3 \mathrm{~L} \times 6 \mathrm{~L} \times 3 \mathrm{R}$ amino acid signature required for the interaction of MYB transcription factors with bHLH transcription factors [41], and the $\mathrm{S}$ residue required for activation of GL2 [42], are full conserved in all the five OsGL1s (Fig. S1); The first 97 amino acids region in GL1 that is required for its interaction with GL3 [9], is highly conserved in both OsGL1A and OsGL1B (Fig. S2); and the 25 amino acids region in TTG1 that is required for its interaction with GL3 [9], is also highly conserved in OsTTG1A and OsTTG1B (Fig. S3). Second, it has been shown that GL3 functions as a transcription activator, but GL1 and TTG1 do not [41, 44], similar, our results show that OsGL3B activated reporter gene expression in transfected protoplast, but OsGL1E 

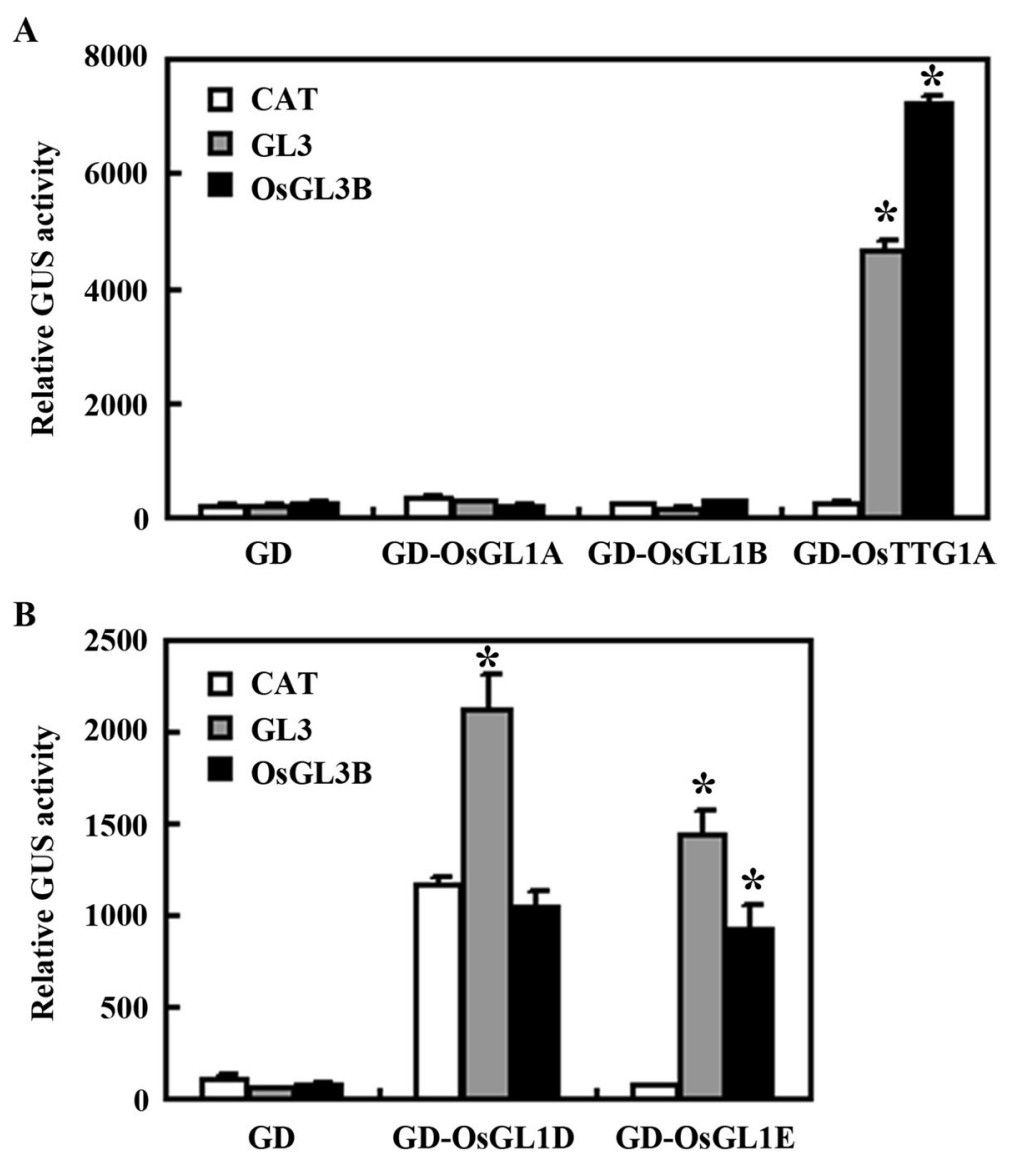

Fig. 5 Interaction of OsGL3B with OsGL1s and OsTTG1A in transfected protoplasts. a Interaction of OsGL3B with OsGL1A, OsGL1B and OsTTG1A. b Interaction of OsGL3B with OsGL1D and OsGL1E. Plasmids of the Gal4:GUS reporter, GD-OsGL1s, and OsGL3B, GL3 or CAT effectors were co-transfected into protoplasts. Cotransfection of the plasmids of GD effector was used as a control. After incubated for $20-22 \mathrm{~h}$ at room temperature in darkness, GUS activities were measured by using a microplate reader. Data represent the mean \pm SD of three biological replicates. *: Significantly different from the CAT control according to the Anova with post-hoc Tukey HSD test (https://astatsa.com/OneWay_Anova_with_Tukey $\mathrm{HSD} /)(P<0.01)$

and OsTTG1 failed to do so (Fig. 3). Third, OsGL3B interacted with OsGL1E and OsTTG1A, respectively (Figs. 3 and 4), indicating that they can form a MBW complex. In addition, OsGL1E and OsTTG1A can interact with GL3 (Figs. 3 and 4), and OsGL3B interacted with GL1 and TTG1 (Fig. 5), suggested that rice homologs are interchangeable with their Arabidopsis MBW complex proteins in forming MBW complexes. Last but not least, ectopic expression of OsTTG1A restored the phenotypes in the ttg1 mutant (Figs. 6, 7 and 8), indicating that OsTTG1A is likely the functional analogue of TTG1 in controlling trichome formation in Arabidopsis. Considering that OsGL1E and OsGL3B are able to form MWB complex with OsTTG1A, it may be worthwhile to examine if they have similar functions with GL1 and GL3, respectively in regulating trichome formation in Arabidopsis. Since OsTTG1A and OsTTG1B show high amino acid sequence similarly, it will be of interest to examine if
OsTTG1B also have similar functions. Considering that OsGL3A is closely related to OsGL3B, it will be also of interest to examine if OsGL3A may form MBW complex with OsGL1s and OsTTG1A.

The MBW complex formed by GL1, GL3/EGL3 and TTG1 regulates trichome initiation via activating GL2 $[3-5,7]$, but the same MBW complex can also activate the R3 MYB trichome initiation inhibitor genes [17-22]. Even though our previously study showed that OsTCL1 can regulate trichome initiation in Arabidopsis, but it can not in rice [40], considering that trichome initiation in rice can be regulated by OsWOX3B, a homolog of GL2 [33-35], it is worthwhile to examine if MBW complex formed by OsGL1E, OsGL3B and OsTTG1A is able to regulate trichome initiation in rice via activating OsWOX3B.

As discussed above, some of our experiments support that rice homologs and the Arabidopsis MBW 
$\mathbf{A}$

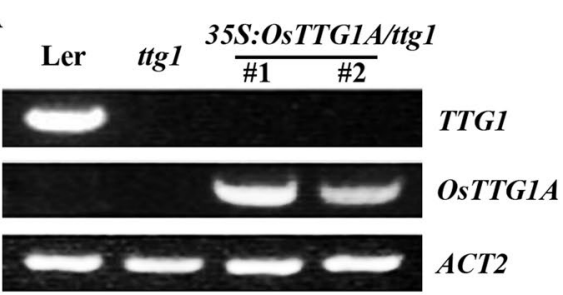

B
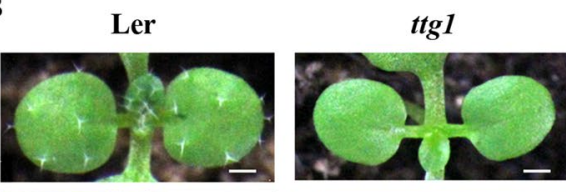

35S:OsTTG1A/ttg1 \#1 35S:OsTTG1A/ttg1 \#2
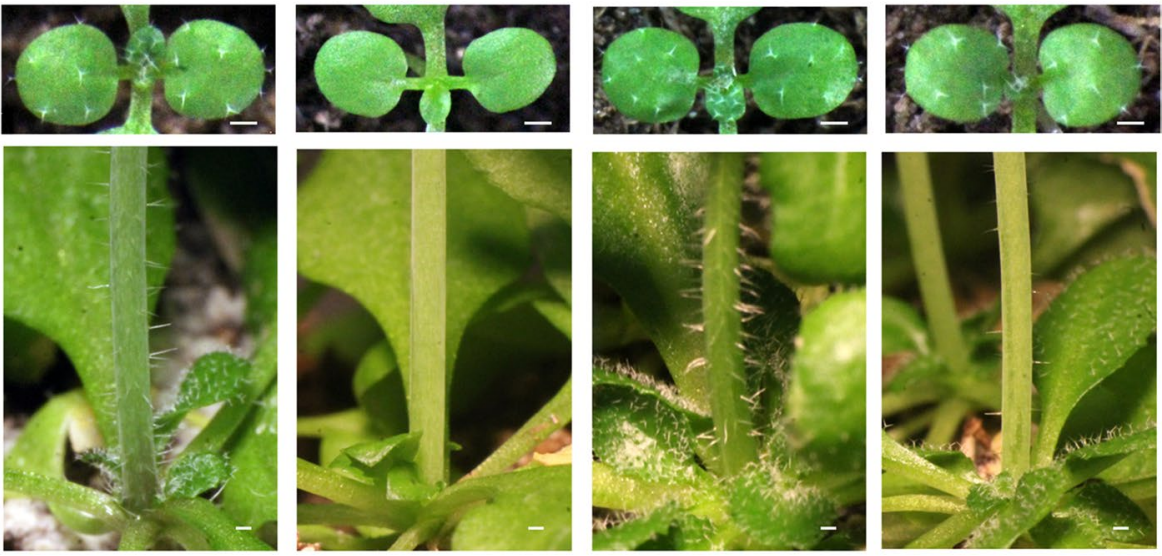

C

\begin{tabular}{cccccc}
\hline \multirow{2}{*}{ Genotype } & Leaf position $\mathbf{1 / 2}$ & $\mathbf{3}$ & $\mathbf{4}$ & $\mathbf{5}$ & $\mathbf{6}$ \\
\cline { 2 - 6 } & $8.2 \pm 1.2$ & $22.3 \pm 2.9$ & $28.8 \pm 3.1$ & $35.8 \pm 4.1$ & $45.6 \pm 4.6$ \\
Ler & 0 & 0 & 0 & 0 & 0 \\
ttg1 & $8.5 \pm 0.8$ & $25.4 \pm 2.7$ & $32.3 \pm 3.8$ & $38.4 \pm 4.7$ & $49.8 \pm 5.4$ \\
35S:OSTTG1A/ttg1 \#1 & $6.7 \pm 1.1$ & $15.2 \pm 2.1$ & $21.5 \pm 2.9$ & $28.3 \pm 3.2$ & $37.4 \pm 3.8$ \\
35S:OSTTG1A/ttg1 \#2 & &
\end{tabular}

Fig. 6 Ectopic expression of OsTTG1A restored trichome phenotype in the $\operatorname{ttg} 1$ mutants. a Expression of TTG1 and OsTTG1A in the Ler wild type, the $t$ tg 1 mutant and the 35 S:OsTTG1/ttg 1 transgenic plants. RNA was isolated from 10-day-old seedlings, and RT-PCR was used to examine the expression of TTG1 and OSTTG1A. The expression of ACT2 was used as a control. b Trichome formation in leaves (up panel) and stems (lower panel) of the Ler wild type, the $\operatorname{ttg} 1$ mutant and 35S:OsTTG1/ttg1 transgenic plants. Bar, $1 \mathrm{~mm}$. c Number of leaf trichomes in the Ler wild-type, the ttg 1 mutant and 35S:OSTTG1/ttg1 transgenic plants. Data represent the mean \pm SD of 12 plants

complex proteins may have conserved functions, but some others also suggest that they may also have nonconserved functions. First, OsGL1A and OsGL1B shared higher amino acid sequence identity and similarity with GL1 when compared with OsGL1D and OsGL1E (Fig. S1), however, they can not interact with GL3 or OsGL3B (Figs. 3 and 4). Considering that the $[D / E] L \times 2[R / K] \times 3 L \times 6 L \times 3 R$ amino acid signature and the $S$ residue required are conserved in all the five OsGL1s (Fig. S1), it is very likely the some other amino acid residues may also be critical for the interaction of OsGL1s/GL1 with GL3/OsGL3B. According to the amino acid sequence alignment, in the R3
MYB domain of OsGL1A and OsGL1B, there are only a few amino acids are different from these in GL1 and OsGL1E, it will be interest to examine if any of them may be critical for the interaction of OsGL1s/GL1 with GL3/OsGL3B. Second, unlike GL1, OsGL1D functions as a transcription activator (Fig. 3). It is unlikely that OsGL1D contains an activation domain, whereas other OsGL1s do not, since previously we have shown that the C-terminal domain of GL1 confer transcriptional activation activities, but as a whole, GL1 does not function as a transcription activator [44]. Therefore, it is worthwhile to figure out why OsGL1D as a whole, is able to show its transcriptional activation 
A
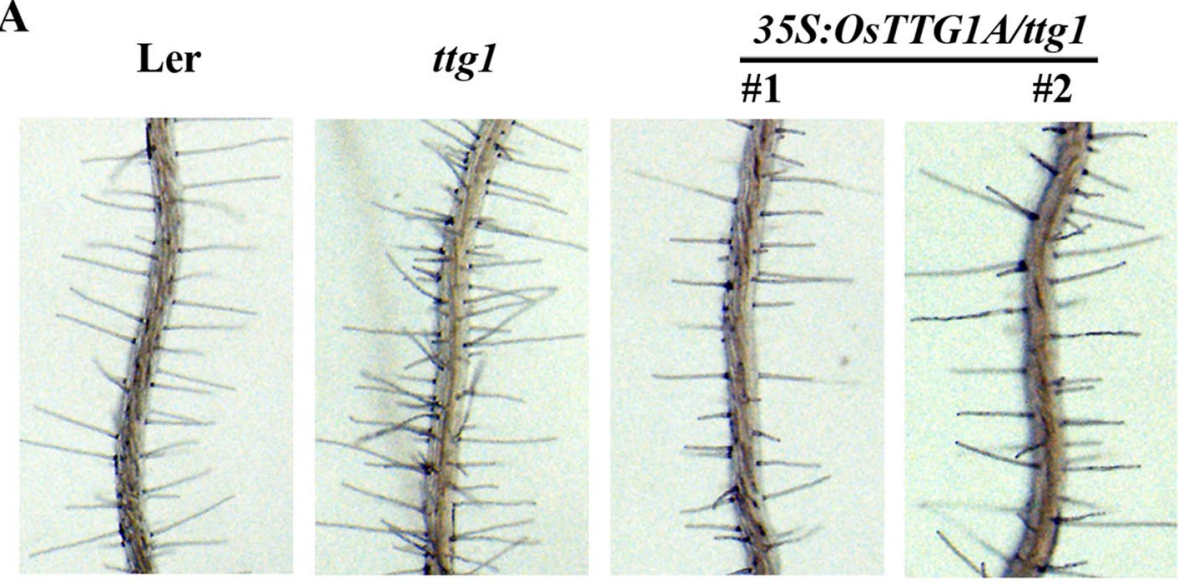

B

\begin{tabular}{cc}
\hline Genotype & Number of root hairs per $\mathbf{m m}$ \\
\hline Ler & $37.0 \pm 4.4$ \\
ttg1 & $57.0 \pm 8.1$ \\
35S:OSTTG1A/ttg1 \#1 & $30.3 \pm 5.5$ \\
35S:OsTTG1A/ttg1 \#2 & $34.8 \pm 4.8$ \\
\hline
\end{tabular}

Fig. 7 Ectopic expression of OsTTG1A restored root hair phenotype in the ttg1 mutants. a Root hair formation in the Ler wild-type, the ttg 1 mutant and 35S:OsTTG1/ttg1 transgenic plants. b Number of root hairs in the Ler wild-type, the ttg1 mutant and 35S:OsTTG1/ttg1 transgenic plants. Data represent the mean \pm SD of at least 15 seedlings

activities, whereas GL1 and other OsGL1s can not. Third, OsGL1D interacted with GL3, but not OsGL3 (Figs. 3 and 4). Considering that OsGL1D is closely related to the OsGL1E (Fig. 1), it will also be interesting to examine why OsGL1D can interact with GL3 but not OsGL3B.

\section{Conclusions}

Our results show that OsGL1E, OsGL3B and OsTTG1A are able to form a MBW complex, and they are interchangeable with the Arabidopsis MBW complex proteins in forming MBW complexes. Further more, OsTTG1A is also interchangeable with TTG1 in regulating trichome initiation in Arabidopsis. Our results also show that OsGL1D is a transcription activator, and it can interact with GL3, but not OsGL3B. These results suggest that rice homologs and the Arabidopsis MBW complex proteins have conserved and non-conserved functions.

\section{Methods}

\section{Bioinformatics analysis}

Identification of GL1 and GL3 homologs in rice (Oryza sativa), including OsGL1A, OsGL1B, OsGL1C, OsGL3A, OsGL3B, OsGL3C, OsTTG1A, and OsTTG1B has been described previously [40]. OsGL1D and OsGL1E were identified as potentially interaction proteins of OsGL3B on STRING (https://string-db.org/).

Full-length amino acid sequences were used for phylogenetic analysis and sequence alignment. Phylogenetic analysis was performed on Phylogeny (www. phylogeny.fr) by using "One Click" mode with default settings. The analysis was performed by selecting "One Click" from the tab "Phylogeny Analysis" on the website, and then inputting the amino acid sequences as promoted. The details setting of "One Click" mode can be viewed by clicking "Overview" after the "One Click" mode was selected. Sequence alignment was generated by using BioEdit. Percentage of amino acid identity and similarity were calculated by using MatGAT (v2.02) [47]. 

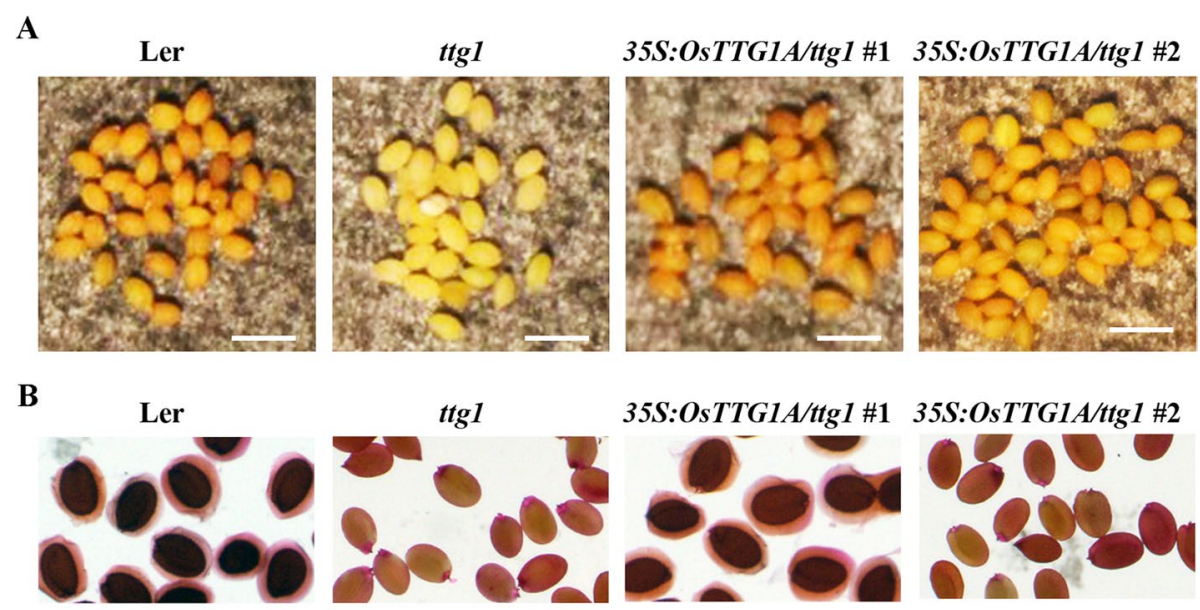

35S:OsTTG1A/ttg1 \#1 35S:OsTTG1A/ttg1 \#2

C
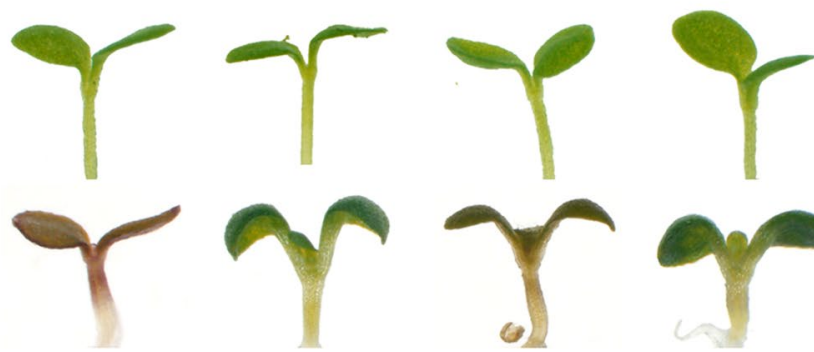

\section{Control}

Ler

$\operatorname{tg} 1$

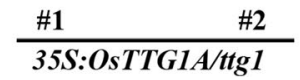

Fig. 8 Ectopic expression of OSTTG1A restored secondary metabolism phenotype in the ttg1 mutants. a Seed coat color in the Ler wild type, the ttg1 mutant and 35S:OsTTG1/ttg1 transgenic plants. Bar, 1 mm. b Mucilage production in the Ler wild type, the $\operatorname{tg} 1$ mutant and 35S:OsTTG1/ttg1 transgenic plants. c Anthocyanin biosynthesis in the Ler wild type, the ttg1 mutant and 35S:OsTTG1/ttg 1 transgenic plants

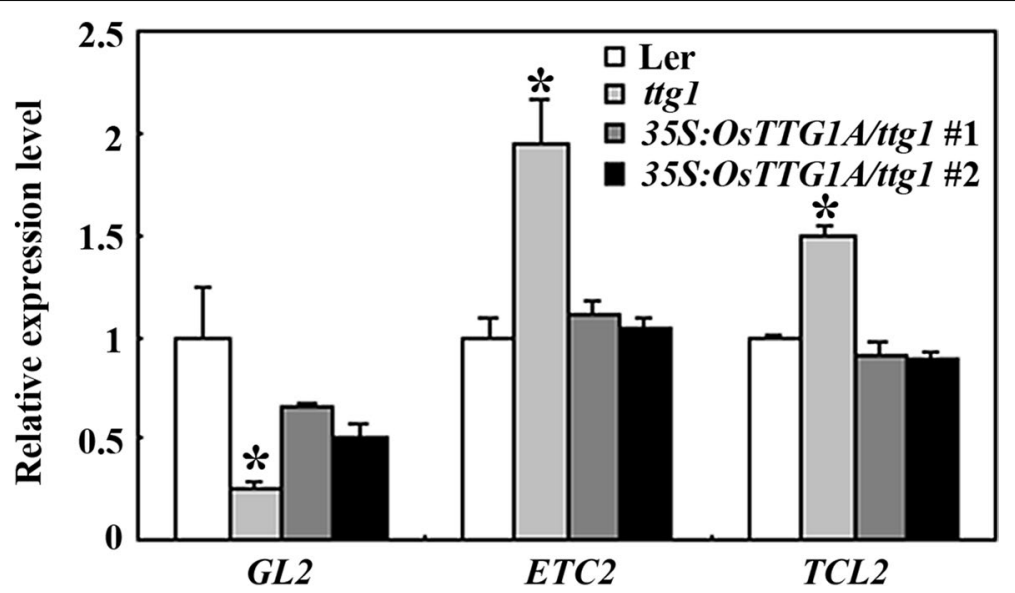

Fig. 9 Expression of trichome formation regulating genes downstream of TTG1 in the 355:OsTTG1A/ttg1 transgenic plants. RNA was isolated from 10-day-old seedlings of the Ler wild type, the $t \operatorname{tg} 1$ mutants, and the 35S:OsTTG1A/ttg1 transgenic plant seedlings and qRT-PCR was used to examine gene expression. ACT2 was used as an inner control, and the expression level of the corresponding genes in the Ler wild type seedings was set as 1. Data represent the mean \pm SD of three biological replicates. * Significantly different from that in the Ler wild type (Student's $t$-test $P<0.05$ ) 


\section{Plant materials and growth conditions}

The Japonica rice variety Nipponbare was used for gene cloning, the Columbia-0 ( $\mathrm{Col})$ Arabidopsis was used for protoplasts isolation, and the Landsberg erecta (Ler) Arabidopsis was used as a control for phenotype analysis. The ttg1 mutant was in the Ler ecotypic background [12].

For trichome phenotypic analysis, protoplast isolation and plant transformation, seeds of indicated Arabidopsis materials were germinated and grown in soil pots as described previously [48]. For root hair phenotypic analysis, seeds were surface-sterilized and sown on solidified 1/2 MS (Murashige and Skoog) medium and grown vertically in a growth room as described previously [40]. More than 15 seedlings for each line were used for trichome and root hair phenotype analysis.

For RNA isolation from Arabidopsis to examine the expression of TTG1 and OsTTG1A, seeds of the Ler wild type, the $\operatorname{ttg} 1$ mutant, and the $35 S$ :OsTTG1A/ttg1 transgenic plants were surface-sterilized and sown on solidified 1/2 MS medium and grown vertically in a growth room as described previously [48]. For each genotype, a mix of at least eight seedlings was used for RNA isolation.

For RNA isolation from rice to clone related genes, seeds of Nipponbare rice were generated and grown in water in a growth room for 10 days. The photoperiod in the growth room was $16 \mathrm{~h}$ light $/ 8 \mathrm{~h}$ dark, the light density was $\sim 120 \mu \mathrm{mol} \mathrm{m} \mathrm{m}^{-2} \mathrm{~s}^{-1}$, and the temperature was $22{ }^{\circ} \mathrm{C}$ for Arabidopsis, and $28^{\circ} \mathrm{C}$ for rice [40]. A mix of five seedlings was used for RNA isolation.

The seeds used for phenotypic analysis and examine the gene expression level were planted and collected in the same condition and same time.

\section{RNA isolation, RT-PCR and qRT-PCR}

Ten-day-old Arabidopsis and rice seedlings were used for RNA isolation. Total RNA was isolated by using an EasyPure ${ }^{\mathrm{TM}}$ Plant RNA Kit (Transgene Biotech) and following the manufacturer's instructions. cDNA was synthesized by using an EasyScript First-Strand DNA Synthesis Super Mix (TransGen Biotech) and following the manufacturer's procedures, and used for RT-PCR and qRT-PCR amplification. For expression analysis of TTG1 and OsTTG1A, the expression of ACTIN2 (ACT2) was used as a control. For expression of GL2 and R3 MYB genes, the expression of $A C T 2$ was used as an inner control.

The primers used for amplification of OsGL1A are 5'-CAACATATGATGGGGAGGTCGCCGTGC-3'and
5'-CAACTTAAGTCATTTCATGGGGAGGCTTCT G-3', for OsGL1B are 5'-CAACATATGATGGGGAGG TCACCG-3'and 5'-CAACTTAAGTCATTTCATTTC CAAGCTTCTG-3', for OsTTG1A are 5'-CAACAT ATGGAGCAGCCCAAGCCG-3' and 5'-CAACTT AAGTCAGACCCTGAGAAGCTGGA-3, for GL1 are 5'-CAACATATGAGAATAAGGAGAAGAGATGA-3' and 5'-CAACTTAAGCTAAAGGCAGTACTCAAC ATC-3', for TTG1 are 5'-CAACATATGATGGATAAT TCAGCTCCAGATTCG-3' and 5'-CAACTTAAG TCAAACTCTAAGGAGCTGCATTTTG-3', and for GL3 are 5'-CAACATATGGCTACCGGACAAAAC AG-3' and 5'-CAAGAGCTCTCAACAGATCCATGC AACCC. Other primers used for RT-PCR have been described previously $[40,48]$.

\section{Constructs}

The nuclear indicator construct NLS-RFP, the reporter construct Gal4:GUS and the effector constructs GD (Gal4 DNA Binding Domain), CAT, GL3, GD-GL3, GD-GL1, GD-TTG1 used for protoplasts transfection have been described previously $[44,49,50]$.

To make the HA (Human influenza hemagglutinin)tagged OsGL3B construct, and GFP (Green fluorescent protein) tagged and/or GD-tagged OsGL1s, OsGL3B and OsTTG1A constructs for protoplast transfection, the full-length ORF (open-reading frame) of the corresponding genes were amplified by RT-PCR using RNA isolated from rice seedlings, or synthesized (for OsGL3B, OsGL1C, OsGL1D and OsGL1E) by Sangon Biotech Co., Ltd, and cloned in-frame with an N-terminal HA, GFP or GD tag and under the control of the double $35 S$ promoter into pUC19 vector [50, 51].

To make 35S:OsTTG1A construct for plant transformation, the HA tagged OsTTG1A construct in $p U C 19$ was digested with NdeI and AflII and subcloned into the binary vector $p P Z P 211$ vector [52].

To generate bait and prey constructs for yeast-twohybrid assays, OsGL3B was cloned into $p G B K T 7$ vector (Oebiotech), and GL1 and TTG1 and their homolog genes in rice were cloned into $p G A D T 7$ vector (Oebiotech).

\section{Plant transformation and transgenic plants selection}

For phenotype rescue experiment, the 35S:OsTTG1A construct was introduced into Agrobacterium tumefaciens GV3101, and used to transform the ttg1 mutant plants by using the floral dip method [53]. The ttg1 mutant plants used for transformation were $\sim 5$-week-old, and have several mature flowers on the main inflorescence.

To select transgenic lines, T1 seeds were sown on $1 / 2$ MS medium containing $50 \mu \mathrm{g} / \mathrm{ml}$ Kanamycin and $100 \mu \mathrm{g} /$ $\mathrm{ml}$ Carbenicillin. More than 20 transgenic plants were obtained, and three trichome bearing $\mathrm{T} 1$ plants were 
chosen to isolate transgenic plants with a single T-DNA insertion locus in T2, and homozygous lines in T3 by germinating on 1/2 MS medium containing $25 \mu \mathrm{g} / \mathrm{ml}$ Kanamycin. Seeds from two homozygous lines were used for phenotypic analysis.

\section{Yeast two-hybrid assays}

Yeast two-hybrid assay was performed by using Yeast Transformation System 2 (Clontech) according to the manufacturer's instructions.

\section{Plasmid DNA isolation, protoplast transfection and GUS activity assay}

Plasmid DNA used for protoplast transfection was isolated using a GoldHi EndoFree Plasmid Maxi Kit (CWBIO) according to the manufacturer's instructions.

Protoplasts were isolated from 50 60 rosette leaves of $3 \sim 4$-week-old Col plants, and transfected with plasmids of the reporter and effector genes by using the procedure described previously [50].

To examine subcellular localization of rice homologs of GL1, GL3 and TTG1, the plasmids of GFP fused constructs and nuclear indicator construct NLS-RFP were cotransfected into protoplasts. The transfected protoplasts were incubated at room temperature and under darkness for $20 \sim 22 \mathrm{~h}$. GFP and RFP florescence were examined and photographed under a florescence microscope.

To examine the transcriptional activity of OsGL3B, plasmids of the reporter gene Gal4:GUS and the effector gene GD-OsGL3B were cotransfected into protoplasts. Cotransfections of $G D$ and GD-GL3 were used as negative and positive controls, respectively. To examine the possible interaction of OsGL3B with GL1 and TTG1 in plant cells, plasmids of the reporter gene Gal4:GUS, the effectors gene GD-GL1 or GD-TTG1, and OsGL3B or $C A T$ were cotransfected into protoplasts. To examine the possible interaction of OsTTG1A or OsGL1s with OsGL3B or GL3, plasmids of the reporter gene Gal4:GUS, the effector genes GD-OsGL1s or GDOsTTG1A, and OsGL3B, GL3 or CAT were cotransfected into protoplasts. Cotransfections of $G D$ were used as controls. The transfected protoplasts were incubated at room temperature and under darkness for $20 \sim 22 \mathrm{~h}$. GUS activities were measured using a microplate reader (Synergy ${ }^{\mathrm{TM}}$ HT, BioTEK). Transfection of each combination contains three biological replicates, and the experiments were repeated at least twice with similar results.

\section{Mucilage production assays}

Seeds were stained and mounted as described previously [54], and mucilage was viewed examined under a Motic $\mathrm{K}$ dissecting microscope. At least 30 seeds were used for the assays.

\section{Anthocyanin biosynthesis assays}

Anthocyanin biosynthesis was assayed as described previously [55], except that $5 \%$ rather than $3 \%$ sucrose was used for the experiment.

\section{Microscopy}

GFP fluorescence in transfected protoplast was observed and photographs were taken under an Olympus FV1000 confocal microscope. Leaf trichome, root hair, mucilage, anthocyanin and seeds color were examined under a Motic K microscope, and photographs were taken using an EOS 1100D digital camera connected to the microscope. Photographs of stem trichomes were taken using an EOS $1100 \mathrm{D}$ digital camera.

\section{Abbreviations}

Col: Columbia-0; GD: Gal4 DNA Binding Domain; GFP: Green fluorescent protein; HA: Human influenza hemagglutinin; Ler: Landsberg erecta; MBW: MYB-bHLH-WD40; MS: Murashige and skoog; ORF: Open-reading frame; PEG: Polyethyleneglycol; SD-TL: SD-Trp-Leu; SD-TLH: SD-Trp-Leu-His; SD-TLHA: SDTrp-Leu-His-Ade; WT: Wild type.

\section{Supplementary Information}

The online version contains supplementary material available at https://doi. org/10.1186/s12870-021-03035-0.

Additional file 1: Fig. S1 Amino acid sequence alignment of GL1 and OsGL1s. Full-length amino acid sequences of GL1 and OsGL1s were used for sequence alignment by using BioEdit. Identify amino acids are shaded in black, and similar ones in gray. Underlines indicate the R2R3 MYB domain. The conserved amino acid signature $[D / E] L \times 2[R / K] \times 3 L \times 6 L \times 3 R$ that is required for interaction between MYB proteins and R/B-like BHLH transcription factors are indicated by arrowheads. The $\mathrm{S}$ has been shown to be required for the interaction of GL1 with GL3/EGL3 is indicated by star.

Additional file 2: Fig. S2 Amino acid sequence alignment of GL3, EGL3 and OsGL3s.Full-length amino acid sequences of GL3, EGL3 and OsGL3s were used for sequence alignment by using BioEdit. Identity amino acids are shaded in black, and similar ones in gray. Black underlines indicate the HLH domain. Red underlines indicate the 97 amino acid sequence required for the interaction of $G L 3$ with $G L 1$.

Additional file 3: Fig. S3 Amino acid sequence alignment of TTG1 and OsTTG1s.Full-length amino acid sequences of TTG1 and OsTTG1s were used for sequence alignment by using BioEdit. Identity amino acids are shaded in black, and similar ones in gray. Underline indicates the 25 amino acid sequence required for the interaction of TTG1 with $G L 3$.

Additional file 4: Fig. S4 Phylogenetic tree of GL1, MYB23, MYB82, OsGL1s and GL1 homologs from other eight plant species. The entire amino acid sequences of GL1, MYB23, MYB82, OsGL1s and GL1 homologs from the Brassicaceae family plants Brassica rapa, Capsella grandiflora and Capsella rubella, the Fabidae family plant Glycine max, the Malpighiales family plant Populus trichocarpa, and the Panicoideae family plants Zea mays, Setaria italica and Panicum hallii were used for phylogenetic analysis on Phylogeny (www.phylogeny.fr) by using "One Click" mode with default settings. The number above the branch indicates branch support values. Bar indicates branch length.

Additional file 5: Fig. S5 Phylogenetic tree of GL3, EGL3, TT8, OsGL3s and GL3 homologs from other eight plant species. The entire amino acid sequences of GL3, EGL3, TT8, OsGL3s and GL3 homologs from the Brassicaceae family plants Brassica rapa, Capsella grandiflora and Capsella rubella, the Fabidae family plant Glycine max, the Malpighiales family plant Populus trichocarpa, and the Panicoideae family plants Zea mays, Setaria italica and 
Panicum hallii were used for phylogenetic analysis on Phylogeny (www. phylogeny.fr) by using "One Click" mode with default settings. The number above the branch indicates branch support values. Bar indicates branch length.

Additional file 6: Fig. S6. Phylogenetic tree of TTG1, OsTTG1s and TTG1 homologs from other eight plant species. The entire amino acid sequences of TTG1, OSTTG1s and TTG1 homologs from the Brassicaceae family plants Brassica rapa, Capsella grandiflora and Capsella rubella, the Fabidae family plant Glycine max, the Malpighiales family plant Populus trichocarpa, and the Panicoideae family plants Zea mays, Setaria italica and Panicum hallii were used for phylogenetic analysis on Phylogeny (www. phylogeny.fr) by using "One Click" mode with default settings. The number above the branch indicates branch support values. Bar indicates branch length.

\section{Acknowledgements}

We thank all the lab members for their help suggestions.

\section{Authors' contributions}

S.W. conceived the study. K.Z. and S.W. designed the experiments. K.Z., X.W. and Y.W. did the experiments. K.Z. and S.W. analyzed the data and drafted the manuscript. All the authors participated in the revision of the manuscript. The authors read and approved the final manuscript.

\section{Funding}

This research was supported by Young Elite Scientist Sponsorship Program of Jilin Province (181905), Nature Science foundation of Jilin Province (20200201155JC), the National Key R\&D Program of China (2016YFD0101900) and a startup funding from Linyi University (LYDX2019BS039). The funders had no role in study design, data collection and analysis, decision to publish, or preparation of the manuscript.

\section{Availability of data and materials}

All data generated or analyzed during this study are included in this published article.

\section{Declarations}

\section{Ethics approval and consent to participate}

Not applicable.

\section{Consent for publication}

Not applicable.

\section{Competing interests}

The authors declare that they have no competing interests.

\section{Author details}

'Key Laboratory of Soybean Molecular Design Breeding, Northeast Institute of Geography and Agroecology, Chinese Academy of Sciences, Changchun, China. ${ }^{2}$ Key Laboratory of Molecular Epigenetics of MOE, Institute of Genetics and Cytology, Northeast Normal University, Changchun, China. ${ }^{3}$ Laboratory of Plant Molecular Genetics \& Crop Gene Editing, School of Life Sciences, Linyi University, Linyi, China.

Received: 25 November 2020 Accepted: 12 May 2021

Published online: 25 May 2021

\section{References}

1. Mauricio R. Costs of resistance to natural enemies in field populations of the annual plant arabidopsis thaliana. Am Nat. 1998;151:20-8.

2. Szymanski DB, Lloyd AM, Marks MD. Progress in the molecular genetic analysis of trichome initiation and morphogenesis in Arabidopsis. Trends Plant Sci. 2000;5:214-9.
3. Nguyen CT, Tran GB, Nguyen NH. The MYB-bHLH-WDR interferers (MBWi) epigenetically suppress the MBW's targets. Biol Cell. 2019;111:284-91.

4. Schiefelbein J, Huang L, Zheng X. Regulation of epidermal cell fate in Arabidopsis roots: the importance of multiple feedback loops. Front Plant Sci. 2013;5:47.

5. Wang S, Chen J. Regulation of cell fate determination by single-repeat R3 MYB transcription factors in Arabidopsis. Front Plant Sci. 2014;5:133.

6. Wang Z, Yang Z, Li F. Updates on molecular mechanisms in the development of branched trichome in Arabidopsis and nonbranched in cotton. Plant Biotechnol J. 2019;17:1706-22.

7. Chen S, Wang S. GLABRA2, a common regulator for epidermal cell fate determination and anthocyanin biosynthesis in Arabidopsis. Int J Mol Sci. 2019;20:4997.

8. Oppenheimer DG, Herman PL, Shan S, Esch J, Marks MD. A myb gene required for leaf trichome differentiation in Arabidopsis is expressed in stipules. Cell. 1991;67:483-93.

9. Payne $C T$, Zhang F, Lloyd AM. GL3 encodes a bHLH protein that regulates trichome development in Arabidopsis through interaction with GL1 and TTG1. Genetics. 2000;156:1349-62.

10. Zhang F, Gonzalez A, Zhao MZ, Payne CT, Lloyd A. A network of redundant bHLH proteins functions in all TTG1-dependent pathways of Arabidopsis. Development. 2003;130:4859-69.

11. Maes L, Inzé D, Goossens A. Functional specialization of the TRANSPARENT TESTA GLABRA1 network allows differential hormonal control of laminal and marginal trichome initiation in arabidopsis rosette leaves. Plant Physiol. 2008;148:1453-64.

12. Walker AR, Davison PA, Bolognesi-Winfield AC, James CM, Srinivasan $N$, Blundell TL, et al. The TRANSPARENT TESTA GLABRA1 locus, which regulates trichome differentiation and anthocyanin biosynthesis in arabidopsis, encodes a wd40 repeat protein. Plant Cell. 1999;11:1337-49.

13. Kirik V, Lee MM, Wester K, Herrmann U, Zheng Z, Oppenheimer D, et al. Functional diversification of MYB23 and GL1 genes in trichome morphogenesis and initiation. Development. 2005;132:1477-85.

14. Liang G, He H, Li Y, Ai Q, Yu D. MYB82 functions in regulation of trichome development in Arabidopsis. J Exp Bot. 2014;65:3215-23.

15. Rerie WG, Feldmann KA, Marks MD. The GLABRA2 gene encodes a homeo domain protein required for normal trichome development in Arabidopsis. Genes Dev. 1994;8:1388-99.

16. Tian $\mathrm{H}$, Wang S. TRANSPARENT TESTA GLABRA1, a key regulator in plants with multiple roles and multiple function mechanisms. Int J Mol Sci. 2020:21:4881.

17. Wada T, Tachibana T, Shimura Y, Okada K. Epidermal cell differentiation in Arabidopsis determined by a Myb homolog. CPC Science. 1997;277:1113-6.

18. Schnittger A, Folkers U, Schwab B, Jürgens G, Hülskamp M. Generation of a spacing pattern: the role of triptychon in trichome patterning in Arabidopsis. Plant Cell. 1999;11:1105-16.

19. Esch JJ, Chen MA, Hillestad M, Marks MD. Comparison of TRY and the closely related At1g01380 gene in controlling Arabidopsis trichome patterning. Plant J. 2004;40:860-9.

20. Simon M, Lee M, Lin Y, Gish L, Schiefelbein J. Distinct and overlapping roles of single-repeat MYB genes in root epidermal patterning. Dev Biol. 2007;311:566-78.

21. Tominaga R, Iwata M, Sano R, Inoue K, Okada K, Wada T. Arabidopsis CAPRICE-LIKE MYB 3 (CPL3) controls endoreduplication and flowering development in addition to trichome and root hair formation. Development. 2008;135:1335-45.

22. Wang S, Hubbard L, Chang Y, Guo J, Schiefelbein J, Chen J. Comprehensive analysis of single-repeat R3 MYB proteins in epidermal cell patterning and their transcriptional regulation in Arabidopsis. BMC Plant Biol. 2008;8:81.

23. Kirik V, Simon M, Huelskamp M, Schiefelbein J. The enhancer of try and CPCl gene acts redundantly with triptychon and caprice in trichome and root hair cell patterning in Arabidopsis. Dev Biol. 2004;268:506-13.

24. Wang S, Kwak S, Zeng Q, Ellis B, Chen X, Schiefelbein J, et al. Trichomeless 1 regulates trichome patterning by suppressing GLABRA1 in Arabidopsis. Development. 2007;134:3873-82. 
25. Gan L, Xia K, Chen J, Wang S. Functional characterization of TRICHOMELESS2, a new single-repeat R3 MYB transcription factor in the regulation of trichome patterning in Arabidopsis. BMC Plant Biol. 2011;11:176.

26. Hülskamp M, Misŕa S, Jürgens $G$. Genetic dissection of trichome cell development in Arabidopsis. Cell. 1994;76:555-66.

27. Pesch M, Hülskamp M. Creating a two-dimensional pattern de novo during Arabidopsis trichome and root hair initiation. Curr Opin Genet Dev. 2004:14:422-7.

28. Ishida T, Kurata T, Okada K, Wada T. A genetic regulatory network in the development of trichomes and root hairs. Annu Rev Plant Biol. 2008;59:365-86.

29. Gruber MY, Wang S, Ethier S, Holowachuk J, Bonham-Smith PC, Soroka J, et al. "Hairy Canola"-Arabidopsis GL3 induces a dense covering of trichomes on Brassica napus seedlings. Plant Mol Biol. 2006;60:679-98.

30. Wang S, Wang JF, Yu N, Li C, Luo B, Gou J, et al. Control of plant trichome development by a cotton fiber MYB gene. Plant Cell. 2004;16:2323-34

31. Guan X, Li Q, Shan C, Wang S, Mao Y, Wang L, et al. The HD-Zip IV gene GaHOX1 from cotton is a functional homologue of the Arabidopsis GLABRA2. Physiol Plant. 2008;134:174-82.

32. Brueggemann J, Weisshaar B, Sagasser M. A WD40-repeat gene from Malus $x$ domestica is a functional homologue of Arabidopsis thaliana transparent testa glabra1. Plant Cell Rep. 2010;29:285-94.

33. Angeles-Shim R, Asano K, Takashi T, Shim J, Kuroha T, Ayano M, et al. A WUSCHEL-related homeobox 3B gene, depilous (dep), confers glabrousness of rice leaves and glumes. Rice. 2012;5:28.

34. Zhang H, Wu K, Wang Y, Peng Y, Hu F, Wen L, et al. A WUSCHEL-like homeobox gene, OsWOX3B responses to NUDA/GL-1 locus in rice. Rice. 2012;5:30.

35. Sun W, Gao D, Xiong Y, Tang X, Xiao X, Wang C, et al. Hairy leaf 6, an AP2/ ERF transcription factor, interacts with OsWOX3B and regulates trichome formation in rice. Mol Plant. 2017;10:1417-33.

36. Yu N, Cai W, Wang S, Shan C, Wang L, Chen X. Temporal control of trichome distribution by microRNA156-targeted SPL genes in arabidopsis thaliana. Plant Cell. 2010;22:2322-35.

37. Lan T, Zheng Y, Su Z, Yu B, Song H, Zheng X, et al. OsSPL 10, a SBP-box gene, plays a dual role in salt tolerance and trichome formation in rice (Oryza sativa L.). G3-Genes Genomes Genet. 2019;9:4107-14.

38. Ding $Y$, Wang $X$, Su L, Zhai J, Cao S, Zhang D, et al. SDG714, a histone H3K9 methyltransferase, is involved in Tos 17 DNA methylation and transposition in rice. Plant Cell. 2007:19:9-22.

39. Worthen JM, Yamburenko MV, Lim J, Nimchuk ZL, Kieber JJ, Schaller GE. Type-B response regulators of rice play key roles in growth, development and cytokinin signaling. Development. 2019;146:dev174870.

40. Zheng K, Tian H, Hu Q, Guo H, Yang L, Cai L, et al. Ectopic expression of R3 MYB transcription factor gene OsTCL1 in Arabidopsis, but not rice, affects trichome and root hair formation. Sci Rep. 2016;6:19254.

41. Zimmermann IM, Heim MA, Weisshaar B, Uhrig JF. Comprehensive identification of Arabidopsis thaliana MYB transcription factors interacting with R/B-like BHLH proteins. Plant J Cell Mol Biol. 2004;40:22-34.

42. Dai $X$, Zhou L, Zhang WF, Cai L, Guo H, Tian H, et al. A single amino acid substitution in the R3 domain of GLABRA1 leads to inhibition of trichome formation in Arabidopsis without affecting its interaction with GLABRA3. Plant Cell Environ. 2016:39:897-907.

43. Zhao M, Morohashi K, Hatlestad G, Grotewold E, Lloyd A. The TTG1-bHLH-MYB complex controls trichome cell fate and patterning through direct targeting of regulatory loci. Development. 2008;135:1991-9.

44. Wang S, Chen J. Arabidopsis Transient Expression Analysis Reveals that Activation of GLABRA2 May Require Concurrent Binding of GLABRA1 and GLABRA3 to the Promoter of GLABRA2. Plant Cell Physiol. 2008:49:1792-804.

45. Koornneef M. The complex syndrome of ttg mutants. Arabidopsis Inform Serv. 1981;18:45-51.

46. Shirley BW, Kubasek WL, Storz G, Bruggemann E, Koornneef M, Ausubel FM, et al. Analysis of Arabidopsis mutants deficient in flavonoid biosynthesis. Plant J. 1995;8:659-71.
47. Campanella JJ, Bitincka L, Smalley J. MatGAT: An application that generates similarity/identity matrices using protein or DNA sequences. BMC Bioinformatics. 2003:4:29.

48. Tian H, Wang X, Guo H, Cheng Y, Hou C, Chen JG, et al. NTL8 regulates trichome formation in Arabidopsis by directly activating R3 MYB genes TRY and TCL1. Plant Physiol. 2017;174:2363-75.

49. Lee MM, Schiefelbein J. Developmentally distinct MYB genes encode functionally equivalent proteins in Arabidopsis. Development (Cambridge, England). 2001;128:1539-46.

50. Wang S, Tiwari SB, Hagen G, Guilfoyle TJ. Auxin response factor7 restores the expression of auxin-responsive genes in mutant arabidopsis leaf mesophyll protoplasts. Plant Cell. 2005;17:1979-93.

51. Tiwari SB, Wang X, Hagen G, Guilfoyle TJ. AUX/IAA proteins are active repressors, and their stability and activity are modulated by auxin. Plant Cell. 2001;13:2809-22.

52. Hajdukiewicz P, Svab Z, Maliga P. The small, versatile pPZP family of Agrobacteriumbinary vectors for plant transformation. Plant Mol Biol. 1994;25:989-94.

53. Clough SJ, Bent AF. Floral dip: A simplified method for Agrobacteriummediated transformation of Arabidopsis thaliana. Plant J. 1999;16:735-43.

54. Wang S, Barron C, Schiefelbein J, Chen JG. Distinct relationships between GLABRA2 and single-repeat R3 MYB transcription factors in the regulation of trichome and root hair patterning in Arabidopsis. New Phytol. 2010;185:387-400.

55. Wang X, Wang X, Hu Q, Dai X, Tian H, Zheng K, et al. Characterization of an activation-tagged mutant uncovers a role of GLABRA2 in anthocyanin biosynthesis in Arabidopsis. Plant J. 2015;83:300-11.

\section{Publisher's Note}

Springer Nature remains neutral with regard to jurisdictional claims in published maps and institutional affiliations.
Ready to submit your research? Choose BMC and benefit from:

- fast, convenient online submission

- thorough peer review by experienced researchers in your field

- rapid publication on acceptance

- support for research data, including large and complex data types

- gold Open Access which fosters wider collaboration and increased citations

- maximum visibility for your research: over $100 \mathrm{M}$ website views per year

At BMC, research is always in progress.

Learn more biomedcentral.com/submissions 